\title{
Alternative Therapiestrategien chronischer Virushepatitiden
}

\author{
Harald Matthes
}

\author{
Alternative Strategies in the Therapy of Chronic Virus Hepatitis
}

\section{Zusammenfassung}

Konventionelle Therapiestrategien bei chronischer Hepatitis B oder $C$ zeigen nur mäßige Heilungserfolge und sind teilweise mit einer hohen Nebenwirkungsrate verbunden. Ferner stellen absolute und relative Kontraindikationen einer Interferontherapie Limitationen ihres Einsatzes dar.

In vorliegendem Artikel werden alternative Therapien bei chronischen Hepatitiden diskutiert. Ein anthroposophisches Therapiekonzept zeigt in ersten Studien Heilungsraten von bis zu $43 \%$ bei chronischer Hepatitis C auf. Dabei werden das Anthroposophikum Mistelgesamtextrakt (Viscum album L.; Abnobaviscum ${ }^{\circledR}$ ), die Phythopharmaka Solanum lycopersicum und Hepatodoron ${ }^{\circledR}$ neben ordnungstherapeutischen Elementen eingesetzt.

S78 Das Phytopharmakon Glycyrrhizin/Sho-saiko-to aus der chinesischen und japanischen Naturheilkunde führt zu Transaminasenreduktion und weist einen mäßig positiven klinischen Langzeiteffekt bez. Zirrhosebildung und HCC-Entwicklung bei chronischer Hepatitis auf, ohne jedoch zu einer Ausheilung der Hepatitis zu führen. Andere Phytopharmaka wie Silymarin, Phyllantus spec., Colchicin sowie die Substanzen Ursodeoxycholsäure, Vitamin E und Misoprostol werden in ihrem Einfluss auf chronische Hepatitiden diskutiert.

\section{Schlüisselwörter}

Hepatitis · Komplimentärmedizin · anthroposophische Therapie · Phytotherapie · Mistel · Viscum album · Solanum lycopersicum • Hepatodoron · Glycyrrhizin · Sho-saiko-to · Silymarin · Phyllantus · Misoprostol · Ursodeoxycholsäure · Vitamin E

\section{Abstract}

Conventional therapeutic strategies in chronic hepatitis B and C are only moderately successful. Furthermore, the therapy is often related to a high rate of side effects and the use of interferon is limited because of contraindications. In this article alternative therapeutic strategies are discussed. An anthroposophical therapeutic concept shows a sustained response rate of $43 \%$ in preliminary studies. The concept includes herbal drugs (a mistletoe extract, phytotherapeutic Solanum lycopersicum and Hepatodoron $^{\circledR}$ ) and therapeutic elements of life style modification. The traditional Japanese and Chinese medicine uses the herbal drug glycyrrhizin/Sho-saiko-to in therapy of hepatitis B and C. It leads to a reduction of transaminases and to a moderate positive long-term effect concerning cirrhosis and HCC in chronic hepatitis. However, a sustained response has not been described for glycyrrhizin. The relevance of other herbal drugs like silymarin, Phyllantus spec. and Colchicin and drugs like vitamin E, ursodeoxycholic acid or Misoprostol in chronic hepatitis are discussed.

\section{Key words}

Hepatitis · Complementary Medicine · Anthroposophical Therapy · Phytotherapy · Mistletoe · Solanum lycopersicum - Hepatodoron · Glycyrrhizin · Sho-saiko-to · Silymarin · Phyllantus · Misoprostol · Ursodeoxycholic Acid · Vitamin E 
Einleitung

Trotz stetigen Fortschritts bei der Behandlung der Virushepatitiden bleiben die Therapieergebnisse unbefriedigend. Bei der chronischen Hepatitis B (HBV) mit Einsatz von Lamivudin und Interferon kommt es zu Ausheilungsraten von lediglich ca. 20-30\% pro Jahr [1 -6]. Bei der chronischen Hepatitis C (HCV) kommt es mit der Standardtherapie (pegyliertes Interferon und Ribavirin) $\mathrm{zu}$ „Sustained-Response-Raten“ von ca. 60-80\% [7-11], wobei diese Werte keine sog. „intention-to-treat“-Analysen (ITT) darstellen, sondern Daten der regelhaft abgeschlossenen Therapien. Neben einer relativ hohen Abbruchrate bei der Standardtherapie (unerwünschte Wirkungen treten bei 60-100\% der behandelten Patienten auf) stellen auch absolute und relative Kontraindikationen (Autoimmunhepatitis, fortgeschrittene Leberzirrhose, Depressionen) der Standardtherapie bei chronischer HCV mit Interferonen ein Problem dar. Für Non-Responder werden keine Behandlungsalternativen angeboten. Die chronische HCV mit Entwicklung einer Leberzirrhose stellt mittlerweile die häufigste Indikation zur Lebertransplantation in Deutschland dar.

Komplementäre Therapieansätze können begleitend $\mathrm{zu}$ einer konventionellen Therapie mit dem Ziel einer verbesserten Ansprechrate der konventionellen Therapie bzw. einer Symptomenlinderung unter der Therapie gegeben werden oder als alleinige Therapie das Ziel verfolgen, Krankheitsprogression $\mathrm{zu}$ verhindern oder gar Heilung (sustained response) anzustreben.

\section{Alternative/komplementäre Therapiestrategien bei chronischen Virushepatitiden}

\section{Anthroposophisches Therapiekonzept}

In den letzten Jahren wurde in der anthroposophischen Medizin ein holistisches Konzept zur Therapie der chronischen Virushepatitiden entwickelt und systematisch untersucht. In diesem Konzept wird eine kombinierte medikamentöse Therapie zusammen mit ordnungstherapeutischen Komponenten wie Bewegungstherapie (Heileurythmie), künstlerischen Therapien (Mal- und Musiktherapie sowie Plastizieren), Ernährungstherapie, äußeren Anwendungen (z.B. Schafgarbenleberwickel) und psychoedukativer Therapie (Gesprächstherapie, Life-Style Modification, Biografiearbeit) angewendet. Essenzielle medikamentöse Bestandteile des anthroposophischen Therapiekonzepts stellen subkutan applizierte Mistelgesamtextrakte (Viscum album L.) in Kombination mit oral verabreichtem Solanum-lycopersicum-Extrakt und dem Präparat Hepatodoron ${ }^{\circledR}$ dar.

Mistelgesamtextrakte besitzen einen immunstimulierenden/ immunmodulierenden Effekt (Übersicht bei [12]). Dabei kommt den einzelnen Mistelbestandteilen in ihrer Kombination ein überadditiver Effekt zu, so dass sie nicht vergleichbar mit Einzelkomponenten sind (z.B. Mistellektin-I-Gabe). Neben einer immunstimulierenden/immunmodulierenden Komponente besitzen Mistelgesamtextrakte auch einen Apoptose induzierenden Effekt (Übersicht bei [13], [14-18]). Für eine Viruselimination bei chronischer Virushepatitis kommt der T-Zellaktivierung (Übersicht bei [19]) wie auch dem Apoptosemechanismus eine hohe Bedeutung zu [20-25].
Das Phytopharmakon Solanum lycopersicum zeigt in In-vitro-Experimenten an HepG2-Zellen einen proliferationshemmenden Effekt ab einer Konzentration $10^{-6}$ (=D6) sowie eine starke Apoptoseinduktion (im Annexin-V- und Caspase-8-/FLICE-Test). Dieser Apoptoseinduktion mittels Caspase-8-Aktivierung kommt ebenfalls eine hohe Bedeutung $\mathrm{zu}$, da bekannt ist, dass das HCV eine Apoptosehemmung durch Caspaseinhibition bedingt und sich damit der intrazellulären Elimination entzieht [26-28].

Hepatodoron $^{\circledR}$ ist ein spezifisches Anthroposophikum mit einem allgemeinen leberregeneratorischen Effekt. Es wird aus getrockneten Blättern der Walderdbeere (Fragaria vesca) und der Weinrebe (Vitis vinifera) in einem spezifischen phamazeutischen Prozess hergestellt.

Eine erste Pilotstudie zeigte 1997 an 21 Patienten mit chronischer Hepatitis $C$ unter einer einjährigen Therapie mit dem Mistelgesamtextrakt Abnobaviscum ${ }^{\circledR}$ Quercus Stufe 6, 1 Amp. $3 \times$ wöchentlich $(\mathrm{d} 1,3,5)$ s.c. appliziert und Solanum lycopersicum äthanol. Decoctum aquosa D4 $3 \times 15$ Tropfen und Hepatodoron ${ }^{\circledR} 3 \times 2$ Tbl./d Heilungsraten von $43 \%$ (9 von $21 \mathrm{~Pa}$ tienten) $[29,30]$. Studien mit alleiniger Anwendung einer immunstimulierenden/immunmodulierenden Therapie mittels Mistelgesamtextrakt waren weniger erfolgreich [31,32].

In einer nachfolgenden Beobachtungsstudie an 47 Patienten, die mindestens 12 Monate Abnobaviscum ${ }^{\circledR}$ Aceris oder Quercus Stufe 4 bis 10 (meist Stufe 5 oder 6) 1 Amp. s.c., Solanum lycopersicum Herba $\mathrm{D}_{4-6}$ (Fa. Weleda) $3 \times 2$ Tbl./d und Hepatodoron ${ }^{\circledR}$ $3 \times 2$ Tbl./d erhielten, jedoch keine ordnungstherapeutischen $\mathrm{Zu}$ satztherapien wie oben beschrieben, zeigte sich eine Ausheilungsrate von $17 \%(8 / 47 ; 2 \times$ Genotyp $1 ; 2 \times$ Genotyp 2 und $4 \times$ Genotyp 3 ). Weitere $42 \%$ (20/47) der Patienten zeigten unter der Therapie eine Senkung der Transaminasen um $>50 \%$ des Ausgangswerts und eine Viruslastsenkung um den Faktor 1000 vom Ausgangsbefund. Bei 40\% (19/47) der behandelten Patienten waren keine signifikanten laborchemischen Veränderungen der Transaminasen und der Viruslast erkennbar [33,34]. Mehr als 50\% der in die Beobachtungsstudie eingeschlossenen Patienten mit chronischer Hepatitis $C$ hatten eine Interferon/Ribavirintherapie bereits erfolglos durchgeführt. Mehr als $80 \%$ der behandelten Patienten fühlten sich unter der Therapie mit Mistelgesamtextrakt (Abnobaviscum $^{\circledR}$, Solanum lycopersicum und Hepatodoron ${ }^{\circledR}$ ) in ihrem Allgemeinbefinden sowie in ihrer Leistungsfähigkeit deutlich verbessert. Auch allgemeine Symptome wie Müdigkeit, Antriebslosigkeit, Völle- und Druckgefühl im Oberbauch verbesserten sich unter der Therapie bei 77\% der behandelten Patienten.

14 der 47 Patienten unterzogen sich vor und nach der durchgeführten Therapie einer Leberbiopsie. Bei allen 3 Patienten, die eine Ausheilung unter der Therapie hatten und sich einer Leberbiopsie unterzogen, kam es auch histomorphologisch zu einer Ausheilung der entzündlichen Aktivität. In den weiteren 11 Fällen ohne Ausheilung war in allen Fällen eine Verbesserung des modifizierten HAI-Scores nach Therapie gegeben $(\mathrm{p}<0,05)$.

Abgesehenen von der (erwünschten) lokalen Rötung an der Einstichstelle der subkutan applizierten Mistelgesamtextrakte als Ausdruck einer lokalen Immunreaktion nach 12-48 Stunden mit kleiner Protuberanz und Induration mit einem max. Durchmesser von $3 \mathrm{~cm}$ kam es nur bei einer Patientin zu einer allergi- 
schen Hautreaktion (Exanthem) durch den Mistelgesamtextrakt. Bei 4\% (2/47) der Patienten zeigte das Präparat Solanum lycopersicum Herba Tbl. anfänglich leichte Übelkeit/Unwohlsein, welche nach Reduktion der Dosis verschwand $(3 \times 1$ Tbl. D4).

Eine derzeit laufende vierarmige Beobachtungsstudie in Berlin zum anthroposophischen Therapiekonzept der Hepatitis C soll weiteren Aufschluss über Therapiemöglichkeiten der chronischen HCV-Infektion sowie eine Therapieoptimierung erbringen.

Derzeit kann eine Kombinationstherapie mit dem Mistelgesamtextrakt Abnobaviscum ${ }^{\circledast}$ aceris, Solanum lycopersicum Herba ${ }^{\circledR}$ und Hepatodoron ${ }^{\circledR}$ im Rahmen des o.g. Therapiekonzeptes als mögliche alternative Behandlungsoption bei Non-Respondern der konventionellen Therapie mit Interferon/Ribavirin oder absoluten wie relativen Kontraindikationen einer Interferontherapie gelten. Dabei stellt jedoch, bezogen auf das Therapieziel einer Ausheilung mit ca. $20 \%$, die komplementärmedizinische Therapieoption eine (noch) unbefriedigende Alternative zur Standardtherapie mit Interferon und Ribavirin dar und ist damit nur als Reservetherapieoption anzusehen.

Als derzeitig optimiertes medikamentöses Behandlungsschema kann folgende Empfehlung gegeben werden:

Beginn der Therapie mit Abnobaviscum ${ }^{\circledR}$ aceris Stufe 6 und 5 (d1 Stufe 6 s.c.; d2, 5 Stufe 5 s.c.; q7) sowie Hepatodoron ${ }^{\circledR} 3 \times 2$ Tbl./d. Nach 4 Wochen der Therapie Hinzunahme von Solanum lycopersicum Herba $\mathrm{D}_{4}$ (Fa. Weleda) $3 \times 2$ Tbl./d. Stellt sich unter der Therapie keine signifikante Reduktion der Transaminasen und der Viruslast nach 12 Wochen ein, sollte die Misteldosis erhöht werden (z.B. d1 Stufe 5, d2, 5 Stufe 4; q1Woche). Spricht die Therapie auch nach 8 Wochen nicht gut an, sollte der Wirtsbaum des Mistelgesamtextraktes gewechselt werden (z.B: Abnobavis$\operatorname{cum}^{\circledR}$ fraxini oder pini). Die Wirtsbaumwechsel beinhalten unterschiedliche Mistelgesamtextrakte mit hohem Mistellektingehalt I (fraxini) oder fast fehlendem Mistellektin I (pini). Dies führt zu unterschiedlichen Aktivitätsmustern der T-Zell-Stimulation (TH1/TH2) und kann damit bei einem schlechten Ansprechen auf die vorherige Therapie den Verlauf günstig beeinflussen (Übersicht bei [35]).

Weitere phytotherapeutische Optionen bei Virushepatitiden (HBV und $\mathrm{HCV}$ )

Die im Folgenden abgehandelten phytotherapeutischen Substanzen werden unter den Gesichtspunkten einer antifibrotischen, antioxidativen sowie einer antiinflammatorischen und immunmodulierenden sowie auch leberregenerierenden Eigenschaft eingesetzt, ohne dass sie jedoch (meist) wirksame antivirale Eigenschaften besitzen und zu einer Ausheilung führen. Sie werden daher i.S. einer komplementären (= ergänzenden) Medikation oder nach erfolgloser konventioneller antiviraler Therapie mit dem Ziel einer Verhinderung bzw. Verlangsamung eines Leberumbaus zur Zirrhose eingesetzt.

\section{Glycyrrhizin/Sho-saiko-to (TJ-9; xiao-chai-hu-tang)}

Glycyrrhizin wird seit mehr als 20 Jahren in der japanischen Medizin (Sho-saiko-to) bei Hepatitis B und C eingesetzt. Es stellt neben Baicalin und Baicalein auch die Hauptkomponente in dem chinesischen Pflanzenextrakt (TJ-9 = xiao-chai-hu-tang) dar, der in der traditionell chinesischen Medizin (TCM) bei Hepatitiden eingesetzt wird. Glycyrrhizin führt zu einer Senkung der Transaminasen [36 - 40] und zu einer Verbesserung der Leberhistologie [36,41]. Tierexperimentell wurden ein antifibrotischer Effekt von Glycyrrhizin auf die Leber [42] wie auch ein immunmodulierender Effekt $[43,44]$ gezeigt. Ob dieser auch klinisch bei HBV und HCV besteht, wird vermutet $[36,37]$, muss jedoch durch prospektive Studien noch evaluiert werden. Bei einer Langzeittherapie mit Glycyrrhizin wird auch ein HCC-präventiver Effekt postuliert [41]. In der Behandlung der HBV wurde Glycyrrhizin auch in Kombination mit Lamivudin erfolgreich eingesetzt [45]. Glycyrrhizin wird üblicherweise dreimal wöchentlich intravenös appliziert. Als unerwünschte Wirkungen bei Glycyrrhizinanwendung sind ein Pseudoaldosteronismus [46 - 48] und eine hypokaliämische Myopathie [49] beschrieben worden.

\section{Silymarin}

Tierexperimentelle Untersuchungen belegen einen antifibrotischen Effekt von Silymarin auf die Leber bei (chemisch induzierter) Hepatitis [50]. Klinisch ist ein Überlebensvorteil bei alkoholischer Leberzirrhose durch Silymaringabe gezeigt worden [51]. Bei viraler Hepatitis wurde Silymarin bisher lediglich in einer Pilotstudie untersucht, die zu einem positiven Ergebnis kam [52]. Weitere Studien müssen folgen, um eine allgemeine Empfehlung für Silymarin bei viraler Hepatitis geben zu können.

\section{Phyllantus spec.}

Phyllanthus spec. (Euphorbiaceae) ist eine häufig vorkommende tropische und subtropische Pflanze und als Heilmittel in der Volksmedizin dieser Länder weit verbreitet. Bei HBV wird sie häufig eingesetzt. Nur in einer Übersichtsarbeit von Calixto [53] wird ein positiver Effekt bei HBV beschrieben. Andere Autoren kommen zu negativen oder sehr schwachen positiven Ergebnissen dieser Pflanzenextrakte bei viraler Hepatitis [54-58].

\section{Weitere Substanzen}

Misoprostol und andere Prostaglandinanaloga wurden als Protektoren einer Leberschädigung beschrieben. Flisiak beschreibt einen Transaminasenabfall in einer klinischen Studie bei Misoprostoleinnahme bei chronischer HBV $[59,60]$. Ob damit auch das klinische Outcome beeinflusst wird, ist offen.

Weitere Therapeutika wie Colchicin, Ursodeoxycholsäure und Vitamin E werden in ihrem Einsatz bei Hepatitiden nur durch präliminäre Daten gestützt. Für Colchicin zeigte Floreani [61] in einer Pilotstudie einen anitiviralen Effekt durch Mitoseinhibition der Hepatozyten. Ursodeoxycholsäure kommt ein zytoprotektiver Effekt zu [62], es wurde in einzelnen Kombinationstherapiestudien mit Interferon [63] bzw. Glycyrrhizin [64] bei Hepatitiden eingesetzt und zeigte einen fraglich positiven Effekt. Ob ein antioxidativer Effekt durch Vitamin-E-Gabe bei Hepatitiden einen positiven klinischen Effekt besitzt, ist ebenfalls offen $[38,65]$.

Zwei neuere Cochrane-Reviews aus dem Jahre 2001 kommen anhand der derzeitigen Datenlage zur Wirksamkeit von Phytotherapeutika bei HCV [66] und von TCM bei HBV [58] zu negativen Ergebnissen. 


\section{Literatur}

${ }^{1}$ Malik AH, Lee WM. Chronic hepatitis B virus infection: treatment strattegies for the next millennium. Ann Intern Med 2000; 132 (9): $723-731$

2 van Nunen $A B$, Janssen HL, Wolters LM, Niesters HG, de Man RA, Schalm SW. Is combination therapy with lamivudine and interferonalpha superior to monotherapy with either drug? Antiviral Res 2001; 52 (2): $139-146$

${ }^{3}$ Hagmeyer KO, Pan YY. Role of lamivudine in the treatment of chronic hepatitis B virus infection. Ann Pharmacother 1999; 33 (10): $1104-1112$

${ }^{4}$ Hoofnagle JH. Therapy of viral hepatitis. Digestion 1998; 59 (5): $563-578$

${ }^{5}$ Jarvis B, Faulds D. Lamivudine. A review of its therapeutic potential in chronic hepatitis B. Drugs 1999; 58 (1): 101 - 141

${ }^{6}$ Rizzetto M. Therapy of chronic viral hepatitis: a critical view. Ital J Gastroenterol Hepatol 1999; 31 (8): 781 - 793

${ }^{7}$ Gordon SC. Treatment of viral hepatitis - 2001. Ann Med 2001; 33 (6): $385-390$

8 Brown PJ, Neuman MG. Digestive Disease Week 2000 conference report: New treatments for chronic hepatitis C. Can J Clin Pharmacol 2001; 8 (2): $67-71$

${ }^{9}$ Perry CM, Jarvis B. Peginterferon-alpha-2a (40kD): A Review of its Use in the Management of Chronic Hepatitis C. Drugs 2001; 61 (15): $2263-2288$

10 Shiffman ML. Pegylated interferons: what role will they play in the treatment of chronic hepatitis c? Curr Gastroenterol Rep 2001; 3 (1): $30-37$

${ }^{11}$ Manns MP, Cornberg M, Wedemeyer H. Current and future treatment of hepatitis C. Indian J Gastroenterol 2001; 20 (Suppl 1): C47 - 51

12 Berg PA, Stein GM. Einfluss einer Misteltherapie auf die Tumorabwehr - Eine kritische immunologische Analyse. In: Scheer R, Bauer R, Becker H, Berg PA, Fintelmann V (Hrsg). Die Mistel in der Tumortherapie. Essen: KVC Verlag 2001; 95-107

13 Büssing A. Leben und sterben lassen - DNA-stabilisierende und zytotoxische Effekte von Viscum album L.-Extrakten. In: Scheer R, Becker H, Berg PA (Hrsg). Grundlagen der Misteltherapie. Stuttgart: Edition Forschung Hippokrates Verlag 1996; 191 - 196

${ }^{14}$ Bantel H, Engels IH, Voelter W, Schulze-Osthoff K, Wesselborg S. Mistletoe lectin activates caspase-8/FLICE independently of death receptor signaling and enhances anticancer drug-induced apoptosis. Cancer Res 1999; 59 (9): $2083-2090$

15 Büssing A, Suzart K, Bergmann J, Pfuller U, Schietzel M, Schweizer K. Induction of apoptosis in human lymphocytes treated with Viscum album L. is mediated by the mistletoe lectins. Cancer Lett 1996; 99 (1): $59-72$

${ }^{16}$ Büssing A, Suzart K, Schweizer K. Differences in the apoptosis-inducing properties of Viscum album L. extracts. Anticancer Drugs 1997; 8 (Suppl 1): S9-14

17 Büssing A, Stein GM, Pfuller U, Schietzel M. Differential binding of toxic lectins from Viscum album L., ML I and ML III, to human lymphocytes. Anticancer Res 1999; 19 (6B): 5095 - 5099

18 Büssing A, Stein GM, Wagner M et al. Accidental cell death and generation of reactive oxygen intermediates in human lymphocytes induced by thionins from Viscum album L. Eur J Biochem 1999; 262 (1): 79-87

19 Pape GR, Diepobler HM, Gerlach TJ, Jung MC, Grüner NH, Schirren CA. Immunbiologie der Hepatitis-C- Virus-Infektion. In: Häussinger D, Niederau C (Hrsg). Hepatitis C. Vol. 8. Berlin, Wien: Blackwell Wissenschafts-Verlag 2001; 50-63

${ }^{20}$ Hayashi N. Apoptosis in liver disease. Intern Med 1998; 37 (2): $191-192$

${ }^{21}$ Lau JY, Xie X, Lai MM, Wu PC. Apoptosis and viral hepatitis. Semin Liver Dis 1998; 18 (2): 169-176

${ }^{22}$ Nasir A, Arora HS, Kaiser HE. Apoptosis and pathogenesis of viral hepatitis C - an update. In Vivo 2000; 14 (1): 297-300

${ }^{23}$ Tai DI, Tsai SL. Apoptosis in viral hepatitis B and C. Chang Gung Med J 2001; 24 (5): 285-293

${ }^{24}$ Gale MJr, Kwieciszewski B, Dossett M, Nakao H, Katze MG. Antiapoptotic and oncogenic potentials of hepatitis $C$ virus are linked to interferon resistance by viral repression of the PKR protein kinase. J Virol 1999; 73 (8): 6506-6516
${ }^{25}$ Galle PR, Hofmann WJ, Walczak H et al. Involvement of the CD95 (APO-1/Fas) receptor and ligand in liver damage. J Exp Med 1995; 182 (5): $1223-1230$

${ }^{26}$ Matthes B, Mühlenfeld K, Langner A, Matthes H. Untersuchungen zur Toxikologie von Solanum lycopersicum. Zeitschrift für Gastroenterologie 1999; 9: 898

${ }^{27}$ Matthes B. Solanum lycopersicum als Heilpflanze. Der Merkurstab 1999; 52 (Sonderband): $57-67$

${ }^{28}$ Bantel H, Lugering A, Poremba C et al. Caspase activation correlates with the degree of inflammatory liver injury in chronic hepatitis $C$ virus infection. Hepatology 2001; 34 (4 Pt 1): 758-767

${ }^{29}$ Matthes H, Grah C, Biesenthal S. Therapiestudie zur Hepatitis C mit Abnoba viscum und Solanum lycopersicum. Der Merkurstab 1997; Sonderheft Juni 97: 26

${ }^{30}$ Matthes B, Grah C, Biesenthal S, Matthes H. Übersicht von Heilversuchen zur Therapie bei Hepatitis $C$ mit Abnoba viscum und Solanum lycopersicum. Zeitschrift für Gastroenterologie 1998; 36 (8): 760

${ }^{31}$ Huber R, Ludtke R, Klassen M, Muller-Buscher G, Wolff-Vorbeck G Scheer R. Effects of a mistletoe preparation with defined lectin content on chronic hepatitis C: an individually controlled cohort study. Eur J Med Res 2001; 6 (9): 399-405

32 Tusenius KJ, Spoek JM, Kramers CW. Iscador Qu for chronic hepatitis C: an exploratory study. Complement Ther Med 2001; 9: 12 - 16 (1)

33 Matthes H. Aspekte zur Therapie der Hepatitis C. Der Merkurstab 1999; 52 (11): 25-41

${ }^{34}$ Matthes H, Matthes B, Biesenthal S. Survey of unconventionally hepatitis $C$ therapy with an aqueous mistletoe extract and lycopersicon esculentum. Gastroenterology 2000; 118 (5): A

${ }^{35}$ Stein GM, Edlund U, Schaller G, Pfüller U, Büssing A, Schietzel M. Polysacharide und Viscotoxine - Immunologische Wirkung und Interaktionen. In: Scheer R, Bauer R, Becker H, Berg PA, Fintelmann V (Hrsg). Die Mistel in der Tumortherapie. Essen: KVC Verlag 2001; $145-162$

36 van Rossum TG, Vulto AG, de Man RA, Brouwer JT, Schalm SW. Review article: glycyrrhizin as a potential treatment for chronic hepatitis $C$. Aliment Pharmacol Ther 1998; 12 (3): 199-205

37 Sato H, Goto W, Yamamura J et al. Therapeutic basis of glycyrrhizin on chronic hepatitis B. Antiviral Res 1996; 30 (2-3): 171 - 177

${ }^{38}$ Patrick L. Hepatitis C: epidemiology and review of complementary/alternative medicine treatments. Altern Med Rev 1999; 4 (4): 220-238

${ }^{39}$ van Rossum TG, Vulto AG, Hop WC, Schalm SW. Glycyrrhizin-induced reduction of ALT in European patients with chronic hepatitis C. Am J Gastroenterol 2001; 96 (8): 2432 - 2437

${ }^{40}$ Eisenburg J. Treatment of chronic hepatitis B. Part 2: Effect of glycyrrhizic acid on the course of illness. Fortschr Med 1992; 110 (21): 395-398

${ }^{41}$ Arase Y, Ikeda K, Murashima N et al. The long term efficacy of glycyrrhizin in chronic hepatitis C patients. Cancer 1997; 79 (8): 1494- 1500

${ }^{42}$ Park EJ, Ko G, Kim J, Sohn DH. Antifibrotic effects of a polysaccharide extracted from Ganoderma lucidum, glycyrrhizin, and pentoxifylline in rats with cirrhosis induced by biliary obstruction. Biol Pharm Bull 1997; 20 (4): 417-420

${ }^{43}$ Yamashiki M, Nishimura A, Nobori T et al. In vitro effects of sho-saikoto on production of granulocyte colony-stimulating factor by mononuclear cells from patients with chronic hepatitis C. Int J Immunopharmacol 1997; 19 (7): 381 - 385

44 Yamashiki M, Nishimura A, Suzuki H, Sakaguchi S, Kosaka Y. Effects of the Japanese herbal medicine „Sho-saiko-to“ (TJ-9) on in vitro interleukin-10 production by peripheral blood mononuclear cells of patients with chronic hepatitis C. Hepatology 1997; 25 (6): 1390-1397

${ }^{45}$ Matsuo K, Takenaka K, Shimomura $\mathrm{H}$ et al. Lamivudine and glycyrrhizin for treatment of chemotherapy-induced hepatitis B virus (HBV) hepatitis in a chronic HBV carrier with non-Hodgkin lymphoma. Leuk Lymphoma 2001; 41 (1-2): 191 - 195

46 van Rossum TG, de Jong FH, Hop WC, Boomsma F, Schalm SW. „Pseudo-aldosteronism“ induced by intravenous glycyrrhizin treatment of chronic hepatitis C patients. J Gastroenterol Hepatol 2001; 16 (7): $789-795$

${ }^{47}$ Kageyama Y. A case of pseudoaldosteronism induced by glycyrrhizin. Nippon Jinzo Gakkai Shi 1992; 34 (1): 99-102

48 Kageyama Y, Suzuki H, Saruta T. Renin-dependency of glycyrrhizininduced pseudoaldosteronism. Endocrinol Jpn 1991; 38 (1): $103-108$

${ }^{49}$ Shintani S, Murase H, Tsukagoshi H, Shiigai T. Glycyrrhizin (licorice)induced hypokalemic myopathy. Report of 2 cases and review of the literature. Eur Neurol 1992; 32 (1): 44-51 
${ }^{50}$ Schuppan D, Strobel D, Hahn EG. Hepatic fibrosis - therapeutic strategies. Digestion 1998; 59: 385-390

51 Ferenci P, Dragosics B, Dittrich H, Frank H, Benda L, Lachs H, Meryn S, Base W, Schneider B. Randomized controlled trial of silimarin treatment in patients with cirrhosis of the liver. J Hepatol 1989; 9: 105-113

52 Buzzelli G, Moscarella S, Giusti A, Duchini A, Marena C, Lampertico M. A pilot study on the liver protective effect of silybin-phosphatidylcholine complex (IdB1016) in chronic active hepatitis. Int J Clin Pharmacol Ther Toxicol 1993; 31 (9): 456-460

${ }^{53}$ Calixto JB, Santos AR, Cechinel Filho V, Yunes RA. A review of the plants of the genus Phyllanthus: their chemistry, pharmacology, and therapeutic potential. Med Res Rev 1998; 18 (4): 225-258

${ }^{54}$ Liu J, Lin H, McIntosh H. Genus Phyllanthus for chronic hepatitis B virus infection: a systematic review. J Viral Hepat 2001; 8 (5): 358 - 366

${ }^{55}$ Liu J, McIntosh $\mathrm{H}$, Lin $\mathrm{H}$. Chinese medicinal herbs for chronic hepatitis B: a systematic review. Liver 2001; 21 (4): 280-286

56 Thamlikitkul V, Wasuwat S, Kanchanapee P. Efficacy of Phyllanthus amarus for eradication of hepatitis B virus in chronic carriers. J Med Assoc Thai 1991; 74 (9): $381-385$

${ }^{57}$ Liu JP, McIntosh H, Lin H. Chinese medicinal herbs for asymptomatic carriers of hepatitis B virus infection (Cochrane Review). Cochrane Database Syst Rev 2001; 2: CD002231

${ }^{58}$ Liu JP, McIntosh $\mathrm{H}$, Lin H. Chinese medicinal herbs for chronic hepatitis B (Cochrane Review). Cochrane Database Syst Rev 2001; 1: CD001940
${ }^{59}$ Flisiak R, Prokopowicz D. Effect of misoprostol on the course of viral hepatitis B. Hepatogastroenterology 1997; 44 (17): 1419-1425

${ }^{60}$ Flisiak R, Prokopowicz D. Effect of misoprostol on serum beta2-microglobulin in the course of viral hepatitis B. Eur J Gastroenterol Hepatol 1999; 11 (11): $1227-1230$

${ }^{61}$ Floreani A, Lobello S, Brunetto M, Aneloni V, Chiaramonte M. Colchicine in chronic hepatitis B: a pilot study. Aliment Pharmacol Ther 1998; 12 (7): $653-656$

${ }^{62}$ Lirussi F, Okolicsanyi L. Cytoprotection in the nineties: experience with ursodeoxycholic acid and silymarin in chronic liver disease. Acta Physiol Hung 1992; 80 (1-4): 363-367

${ }^{63}$ Fabbri C, Mazzella G, Marchetto S, Pezzoli A. Efficacy of Ursodeoxycholic acid administration as adjuvant therapy together with alfa-interferon for chronic hepatitis C. J Hepatology 1997; 26 (Suppl 1): A179

${ }^{64}$ Tsubota A, Kumada H, Arase $\mathrm{Y}$ et al. Combined ursodeoxycholic acid and glycyrrhizin therapy for chronic hepatitis $C$ virus infection: a randomized controlled trial in 170 patients. Eur J Gastroenterol Hepatol 1999; 11 (10): $1077-1083$

65 Pittschieler K. Oxidative radicals and liver involvement of infants with alpha-1-antitrypsin deficiency. Padiatr Padol 1991; 26 (5): 235-237

${ }^{66}$ Liu JP, Manheimer E, Tsutani K, Gluud C. Medicinal herbs for hepatitis $C$ virus infection (Cochrane Review). Cochrane Database Syst Rev 2001; 4: CD003183 